\title{
Hypnozoites in Plasmodium: do parasites parallel plants?
}

Catherine J. Merrick*

4

Department of Pathology, Cambridge University, Tennis Court Road, Cambridge, CB2 1QP, United

5 Kingdom.

*Correspondence: cim48@cam.ac.uk (C. J. Merrick)

7 Keywords: Plasmodium, malaria, P. vivax, hypnozoites, vernalisation, epigenetics

\section{Abstract}

10 The phenomenon of relapsing malaria has been recognised for centuries. It is caused in

11 humans by the parasite species Plasmodium vivax and ovale, which can arrest growth at an

12 early, asymptomatic stage as hypnozoites inside liver cells. These dormant parasites can

13 remain quiescent for months or years, then reactivate causing symptomatic malaria. The

14 dynamics of hypnozoite dormancy and reactivation are well documented but the molecular

basis remains a complete mystery. Here I observe that the process has striking parallels

with plant vernalisation, whereby plants remain dormant through the winter before

17 flowering in spring. Vernalisation is thoroughly studied in several plant species and its mechanisms are known in exquisite detail. Vernalisation may thus provide a useful framework for interrogating hypnozoite biology. 
Plasmodium hypnozoites: what are they and why do they matter?

Hypnozoites (see Glossary) are the most enigmatic cell types in the complex lifecycle of Plasmodium. In their natural state in the human liver they are small, sparse and difficult to find; in the laboratory they are equally difficult to create and culture. The very existence of a liver stage in the Plasmodium lifecycle (Figure 1) was hypothesised well before its existence was actually proved (which occurred in the 1940s, via voluntary human infection and liver biopsy [1]) and a further four decades then passed before it was finally demonstrated that hepatic $P$. vivax parasites could indeed become hypnozoites [2].

Of the six Plasmodium species that cause malaria in humans, only three are widely believed to form hypnozoites (in fact, direct evidence is sparse in $P$. ovale wallikeri and $P$. ovale curtisi [3]). It is obligatory for all human malaria parasites to pass through a stage of hepatocyte infection before erythrocyte infection, which is the symptomatic and transmissible stage, but it is not obligatory for those hepatic stages to be able to arrest as hypnozoites. Nevertheless, it offers a clear evolutionary advantage. In climates where mosquito transmission - which is indispensable for Plasmodium lifecycles - is available for only a few months per year (due to a brief rainy season or a brief warm summer), the transmission cycle may not be successfully completed before the mosquito season is over. If dormant parasites can remain in the human liver after a single infectious mosquito bite, reactivating only when a new generation of mosquitoes becomes available to pass parasites to new hosts, then the lifecycle can survive despite lengthy seasonal interruptions.

In the current era when malaria elimination is being actively pursued in many countries, it has never been more important to understand hypnozoites and how to kill them. The global malaria burden is dominated by the non-relapsing parasite $P$. falciparum, but the canonical relapsing species, $P$. vivax, is the second most important cause of human 
malaria. P. vivax is responsible for $\sim 3.3 \%$ of all global infections, including $75 \%$ of those in the Americas and $50 \%$ of those in S.E. Asia [4], while P. ovale is a smaller but probably under-reported cause of malaria in Africa. (Indeed, there is growing concern that P. vivax, historically absent from most of Africa, may be on the increase there due to a previously unrecognised ability to infect Duffy-negative erythrocytes [5].) Eliminating $P$. vivax from highly endemic populations could be impossible unless dormant hypnozoites can be killedtermed 'radical cure' [6] - but hypnozoites are not killed by most antimalarial drugs. Only the 8-aminoquinolines primaquine and tafenoquine are effective, and their side-effects make them difficult to administer and can lead to low compliance. New methods to eliminate hypnozoites are urgently needed [7].

\section{Hypnozoite dormancy and reactivation dynamics in $P$. vivax}

The dynamic behaviour of $P$. vivax hypnozoites was clinically documented long before their existence as cells was actually proven. In the early 1900 s this parasite was commonly used to induce high fevers as 'malariotherapy' for syphilis. For this therapy, strains that caused regular, predictable symptomatic bouts of malaria were valued, and therefore carefully curated. This history has been authoritatively reviewed [8] and is summarised here only briefly.

P. vivax strains fall into two main categories (Figure 2A). 'Tropical' strains can relapse within as little as 3 weeks after a symptomatic bout if that bout is promptly cleared with antimalarials (although untreated illness and eventual immunity can suppress such relapses). 'Sub-tropical' or 'temperate' strains relapse only after $\sim 9$ months and may not cause a primary bout of malaria at all, particularly if the inoculum of sporozoites is low, or if the parasite comes from the northern limits of the geographical range [8,9]. Tropical 
strains are mostly found in regions where mosquito transmission is available year-round and sub-tropical strains, where mosquitoes are seasonal [10]. However, humans who do not live in these regions and are artificially infected in any month of the year, or who leave an endemic region after infection, retain the characteristic relapse pattern of the infecting strain. Importantly, this picture of two categories is probably somewhat oversimplified, with modern studies reporting a more complex picture of frequent and variable-interval relapses in endemic regions [11].

The likelihood of a primary bout of malaria after an infectious mosquito bite varies not only with the $P$. vivax strain involved but also with the size of the sporozoite inoculum. With tropical strains, even a few sporozoites usually give a bout of malaria shortly after inoculation, whereas $\geq 1000$ sporozoites of a sub-tropical strain are required; otherwise the primary infection will usually remain silent until a reactivation $\sim 9$ months later $[8,12]$. This gave rise to the hypothesis that hypnozoites form at different default rates: in sub-tropical strains the rate would be 999:1, whereas in tropical strains it would be closer to 1:1. Later on in the course of infection, a larger inoculum will usually result in a longer series of successive relapses and these relapses will occur at shorter, more regular intervals [13-15]. A final intriguing observation is that many fever-inducing illnesses can trigger $P$. vivax relapses [16]. These include $P$. falciparum malaria (which is severe, but not itself relapsing) and bacterial diseases such as typhoid fever. Viral diseases like influenza generally do not have this effect, despite inducing fever (albeit one case report has cited Dengue virus as a potential cause of a $P$. ovale relapse [17]). Hence the hypothesis that malarial bouts, or other systemic inflammatory diseases, can be the stimulus that 'sets the clock' for hypnozoite reactivation $[8,16]$. 
Hypnozoites are unique in the Plasmodium lifecycle. Other stages can also display quiescence - either physiological quiescence, which is seen in circulating gametocytes awaiting a mosquito bite [18] (Figure 1), or induced quiescence seen in erythrocytic parasites when they are treated with the antimalarial drug artemisinin [19]. However, both of these cell types are relatively short-lived and, for gametocytes, the external stimuli that cause reactivation after a mosquito bite are clearly established [20]. By contrast, hypnozoites can persist for months or years and they evidently have an intrinsic, strainspecific reactivation 'clock'. This clock could theoretically be intrinsically timed, stimulusdependent, or a combination of both: its molecular nature is entirely unknown.

The observation that hypnozoites reactivate with predictable timing even after a host has left an endemic region makes it unlikely that hypnozoites can sense seasonal environmental stimuli such as climatic conditions, host nutritional patterns and circadian rhythms that may track with seasons, or direct mosquito-biting of the host. (This latter, remarkably, has been implicated in seasonal rates of conversion to gametocytes in bird malaria parasites $[21,22]$.) Setting aside environmental stimuli, it seems likely that there is instead an intrinsic mechanism, encoded genetically or epigenetically, that slowly builds or decays until each hypnozoite stochastically reactivates. If so, this mechanism must vary between $P$. vivax strains in its strength or longevity, being at 'baseline' in fast-relapsing tropical strains, and perhaps being re-set during the sexual stage in the mosquito.

In fact, the hypnozoite clock probably has additional complexity: certain signals from host physiology may be sensed and integrated into it. If episodes of fever can promote reactivation then hypnozoites can presumably sense an inflammatory environment. $A$ simple heatshock response to elevated body temperature would not suffice because viral 
117 fevers do not trigger relapses; instead the specific cytokine environment of a systemic 118 parasitic or bacterial infection could be sensed [16]. In addition, since the size of the sporozoite inoculum apparently influences the relapse rate, quorum-sensing among hypnozoites could be another signal integrated into the clock. (An alternative, and perhaps simpler, explanation is that large pools of hypnozoites may just be more likely to contain a minority of relatively labile cells, with the fastest-reactivating cell naturally precipitating the first relapse [8]. However, hypnozoites do retain active protein export pathways [23], and circulating erythrocytic parasites are thought to communicate via extracellular vesicles [24], lending plausibility to the idea that hepatic parasites could also communicate.)

\section{The parallel with plants}

Many of the features described above are remarkably similar to the biology of flowering plants, which, like relapsing malaria parasites, can modulate their developmental patterns, remaining in vegetative growth during the cold temperatures and short days of winter before flowering in spring [25]. This is termed vernalisation. in $P$. vivax, there are both fast-cycling and vernalising strains of many plants, from dicotelydons like Arabidopsis to distantly related monocotyledons like wheat. Furthermore, the strength of the vernalisation phenotype varies geographically, with plant strains endemic to northern climes vernalising over longer periods than those from more southern climes - so, like $P$. vivax, the same species can colonise widely different areas of the globe [26].

Secondly, plant strains that are reciprocally transplanted to different latitudes retain their own clock in the new environment [27], showing that vernalisation must be genetically 
or epigenetically encoded. This has in fact been elucidated in great detail, as described below.

Thirdly, like $P$. vivax, plants can integrate several signals, including temperature and day-length, into their intrinsic vernalisation clock, and the clock can be set back by warm clock).

Are these parallels biologically meaningful? Plasmodium parasites have plant-like origins, being evolved from free-living algae [29], but they clearly differ greatly from the eukaryotic life merely have encountered similar evolutionary pressures to respond to seasonal patterns, with similar dormancy and reactivation phenotypes emerging independently as a result? Indeed it may be so, but the molecular basis of the two and one could therefore provide a useful model for studying the other. cereal crops like wheat (Triticum spp.) (Figure 3B). Cold-induced signalling operates however, there is a central MADS-box protein encoded by a gene that bears a slowlyexpression of the master regulator gene during winter, thence affecting the expression of downstream flowering genes. 
by binding to key 'floral integrator' genes like FLOWERING TIME (FT). FLC expression is set

by the balance between repression via histone deacetylation/methylation and

heterochromatinization, versus upregulation by reversal of this chromatin environment - a

process involving the coiled-coil protein FRIGIDA $(F R I)$ which recruits chromatin modifiers

and transcriptional activators [30, 31]. Fast-cycling Arabidopsis strains frequently have null

alleles of $F R I$ or $F L C$. In vernalising strains, by contrast, $F R I$ and $F L C$ expression are high by

default in vegetatively-growing plants, thus repressing flowering [32], but a cold-sensitive

pathway is able to silence $F L C$. This involves thermosensitive components including a

polycomb-like chromatin remodeller VERNALISATION 2 (VRN2) and a plant homeodomain

protein VERNALISATION INSENSITIVE 3 (VIN3). During the cold winter, these slowly enforce

silencing of $F L C$ by stochastically switching off the gene, cell-by-cell with a low probability

per cell. The longer the winter, the more cells will reach a state of low FLC expression and

high expression of flowering genes, so the greater the stimulus to flower when spring

arrives. This requires integration with another signal, lengthening daylight, to confirm that

it is indeed spring. The repressed-FLC situation then pertains throughout summer due to

stable epigenetic marks such as histone methylation being imposed on the FLC locus, until

those marks are erased and expression is reset to a high level in newly formed seeds. FLC is

the major quantitative trait locus (QTL) determining the variable duration of cold exposure

required to trigger flowering, with non-coding variation in the first intron of $F L C$ being responsible for the quantitative trait [30, 31].

Cereal plants also possess conserved flowering genes like $F T$, but a different MADS-

box gene controls them (Figure 3B). VERNALISATION 1 (VRN1), which is not a homolog of 
turn represses $F T$. Vernalisation progressively induces VRN1 expression, ramping up VRN2 repression and hence relieving its repression of $F T$. Day-length signals are then integrated, flowering induced, and high VRN1 expression is maintained throughout flowering via activating rather than repressive histone methylations. Like $F L C, V R N 1$ is a QTL for the length of the vernalisation period - this time via coding SNPs rather than non-coding variation $[33,34]$. Thus, cold-sensing can affect a MADS-box regulator in either direction: in wheat it results in more VRN1 expression, in Arabidopsis it results in less FLC expression. The conserved principle is one of slow epigenetic changes that cumulatively alter expression of a central regulator as winter progresses.

\section{Is vernalisation a good model for hypnozoite biology?}

Almost nothing is yet known about the molecular mechanisms controlling hypnozoites. Unlike plants, they are nearly inaccessible to experimentation because they reside in low numbers in human livers. Within the past decade, seminal work with cultured hypnozoites has begun to change this situation (see Box 1), using primarily the primate model species $P$. cynomolgi, which is closely related to $P$. vivax. Hypnozoite transcriptomes have thus begun to appear but no compelling candidates have emerged for a unique gene controlling dormancy or reactivation. So can a useful model for the control of hypnozoites be built by using the paradigm of plant vernalisation? box family is absent in Plasmodium, which has a restricted set of transcription factors [35, 36]. A central regulator is instead most likely to be found in the APETALA-2 (ApiAP2) family, which contains other master regulators of lifecycle transitions in Plasmodium - some of them, such as the gametocyte regulator $A P 2-G$, being epigenetically controlled $[37,38]$. 
default, depending on whether the putative epigenetic switch more closely resembles that in wheat (VRN1) or Arabidopsis (FLC) (Figure 3C-3D). It is perhaps simplest to imagine a factor for active replication (Figure $3 \mathrm{C}$ ), so $\mathrm{AP2}-\mathrm{H}$ would be on in hepatic and erythrocytic schizonts; off in hypnozoites and perhaps also gametocytes. After passing through a mosquito, $A P 2-H$ would be set to silent in most sporozoites, making hypnozoite formation the default pathway after liver infection. Epigenetic silencing marks on the $A P 2-H$ gene would decay only very slowly, perhaps precipitated by infrequent DNA repair events and histone turnover (pathways for DNA repair and oxidative stress response do remain active in hypnozoites $[23,39,40])$. On average, $\sim 9$ months of epigenetic decay would be required to reach a tipping point for expression of $A P 2-H$ and hence its downstream genes. A fever episode, however, would trigger fast erasure of epigenetic marks - perhaps via extreme stress and DNA damage in an inflammatory environment, or perhaps via specifically induced proteins. This would quickly activate a proportion of hypnozoites and cause a malaria relapse, so after each fever stimulus some hypnozoites would become 'fast cycling'. This is consistent with the observation that sub-tropical strains can remain dormant for 9 months before the first relapse and subsequently become fast-cycling [8] (Figure 2A). Variation in the AP2-H gene (or possibly in its activators or repressors if a more complex signalling cascade were involved) would make this gene in sub-tropical strains much more refractory to activation than the same gene in tropical strains. Thus, hepatic parasites of tropical strains would be prone to escape dormancy immediately after infection, and prone to become fast-cycling, whereas sub-tropical strains would be biased towards dormancy after infection, and generally prone to slow-cycling. 
237 QTLs for the length of dormancy. Characteristic gene variants should exist in tropical and sub-tropical strains. (2) Silencing epigenetic marks should be detectable on AP2-H in hypnozoites. These marks may well be histone methylations, since histone methyltransferase inhibitors can promote reactivation [41]. (3) Null mutants in $A P 2-H$

241 would be inviable (unable to grow), whereas constitutively active versions would never make hypnozoites. Therefore any homolog of $A P 2-H$ in non-relapsing Plasmodium species should be constitutively expressed. (Alternatively, if AP2-H was a growth-silencer as shown in Figure 3D, turned off for reactivation, then null mutants would never make hypnozoites and constitutive versions would be inviable.) (4) This model may be sufficient to explain most of the observed dynamics of hypnozoites, but multicellular plants are more sophisticated: they generate a more 'tuneable' clock by integrating epigenetic switching intriguing to speculate that quorum-sensing between them might modulate reactivation rates. Quorum-sensing could have evolved if, for example, it were advantageous in a heavily infected liver to speed up the epigenetic decay rate, generating many rapid relapses because plenty of hypnozoites would be available for future reactivations if the first did not lead to transmission.

\section{Concluding remarks}

Evidencing this speculative model for hypnozoite biology would not be simple, given the technical challenges of working with hypnozoites (see Outstanding Questions). The simplest route may be to seek ApiAP2 genes, or other transcriptional regulators, that are 
present in $P$. vivax, $P$. ovale and $P$. cynomolgi but absent in non-relapsing species. Genomic studies have indeed pointed to some such genes $[42,43]$. However, a master regulator gene may well be differentially expressed rather than present/absent, so simple genomics would fail to identify it. Nevertheless, characteristic variants in putative regulator gene(s) should be detectable in the genomes of $P$. vivax strains from tropical versus temperate regions (although it would complicate matters if a QTL were on an upstream gene rather than the putative ApiAP2 gene itself). Interestingly, the first Plasmodium virus was recently reported and is apparently unique to $P$. vivax [44]. This viral genome could potentially confer dormancy and reactivation - a characteristic of many viral diseases - but as yet there is no evidence for this.

An alternative route, moving beyond in silico genomics, could be to perform a genetic screen for mutants that cannot form hypnozoites. Unfortunately, genetic tools are lacking in P. vivax, limited in P. cynomolgi, and only one forward genetic screen has ever been achieved in P. falciparum, the most tractable Plasmodium species [45]. It may therefore be more feasible to conduct additional comparative transcriptomic studies and identify candidate genes whose transcription differs in dormant vz. reactivating hypnozoites. Recent advances in single-cell RNA sequencing might even make this feasible could be identified via chromatin immunoprecipitation. within the past decade in hypnozoite culture systems (Box 1), and in genomic sequencing of diverse Plasmodium strains. With these tools, the mysterious molecular biology of hypnozoites could soon be resolved. 
I am grateful to Drs Francis Totanes, Franck Dumetz and Andrew Blagborough for critical reading of this manuscript.

\section{Funding}

This work was supported by an ERC Research grant, 'Plasmocycle' (725126).

\section{References}

292

1. Shortt, H.E. et al. (1948) The pre-erythrocytic stage of human malaria, Plasmodium vivax. Br Med J

293 $1(4550), 547$.

294

2. Krotoski, W.A. et al. (1982) Demonstration of hypnozoites in sporozoite-transmitted Plasmodium vivax infection. Am J Trop Med Hyg 31 (6), 1291-3.

296

3. Richter, J. et al. (2010) What is the evidence for the existence of Plasmodium ovale hypnozoites?

297 Parasitol Res 107 (6), 1285-90.

4. WHO (2019) World Malaria Report 2019.

5. Gunalan, K. et al. (2018) Plasmodium vivax Infections of Duffy-Negative Erythrocytes: Historically Undetected or a Recent Adaptation? Trends Parasitol 34 (5), 420-429.

6. Tatem, A.J. et al. (2010) Ranking of elimination feasibility between malaria-endemic countries. Lancet 376 (9752), 1579-91. vivax: a round table discussion of the APMEN Vivax Working Group. Malar J 16 (1), 141. 297. 

Plasmodium vivax Relapses in Cambodia. mBio 9 (1).

14. Shute, P.G. et al. (1976) A strain of Plasmodium vivax characterized by prolonged incubation: the effect of numbers of sporozoites on the length of the prepatent period. Trans R Soc Trop Med Hyg $70(5-6), 474-81$. 15. Bray, R.S. and Garnham, P.C. (1982) The life-cycle of primate malaria parasites. Br Med Bull 38 (2), 117-22. 16. Shanks, G.D. and White, N.J. (2013) The activation of vivax malaria hypnozoites by infectious diseases. Lancet Infect Dis 13 (10), 900-6. 17. Lupi, O. et al. (2016) Dengue infection as a potential trigger of an imported Plasmodium ovale malaria relapse or a long incubation period in a non-endemic malaria region. Int J Infect Dis 44, 20-4. 18. Yuda, M. et al. (2015) Global transcriptional repression: An initial and essential step for Plasmodium sexual development. Proc Natl Acad Sci U S A 112 (41), 12824-9. 19. Hott, A. et al. (2015) Artemisinin-Resistant Plasmodium falciparum Parasites Exhibit Altered Patterns of Development in Infected Erythrocytes. Antimicrob Agents Chemother 59 (6), 3156-67. 20. Billker, O. et al. (2004) Calcium and a calcium-dependent protein kinase regulate gamete formation and mosquito transmission in a malaria parasite. Cell 117 (4), 503-14. 

prevalence in asymptomatic chronic Plasmodium falciparum infections and correlation with IgE and IgG titers. Infect Immun 80 (6), 2240-6.

22. Cornet, S. et al. (2014) Evolution of plastic transmission strategies in avian malaria. PLoS Pathog 10 (9), e1004308.

23. Bertschi, N.L. et al. (2018) Transcriptomic analysis reveals reduced transcriptional activity in the malaria parasite Plasmodium cynomolgi during progression into dormancy. Elife 7. 24. Mantel, P.Y. and Marti, M. (2014) The role of extracellular vesicles in Plasmodium and other protozoan parasites. Cell Microbiol 16 (3), 344-54. 31.

26. Kenta, T.Y., A.; Onda Y. (2011) Clinal Variation in Flowering Time and Vernalisation Requirement across a 3000-M Altitudinal Range in Perennial Arabidopsis kamchatica Ssp.Kamchatica and Annual Lowland Subspecies Kawasakiana Journal of Ecosystem \& Ecography S6:001.

27. Agren, J. and Schemske, D.W. (2012) Reciprocal transplants demonstrate strong adaptive differentiation of the model organism Arabidopsis thaliana in its native range. New Phytol 194 (4), $1112-22$. Arabidopsis. Nat Commun 9 (1), 639. 29. Moore, R.B. et al. (2008) A photosynthetic alveolate closely related to apicomplexan parasites. Nature 451 (7181), 959-63. 30. Whittaker, C. and Dean, C. (2017) The FLC Locus: A Platform for Discoveries in Epigenetics and Adaptation. Annu Rev Cell Dev Biol 33, 555-575. 31. Bloomer, R.H. and Dean, C. (2017) Fine-tuning timing: natural variation informs the mechanistic basis of the switch to flowering in Arabidopsis thaliana. J Exp Bot 68 (20), 5439-5452. 
32. Gazzani, S. et al. (2003) Analysis of the molecular basis of flowering time variation in Arabidopsis accessions. Plant Physiol 132 (2), 1107-14.

33. Trevaskis, B. et al. (2007) The molecular basis of vernalization-induced flowering in cereals. Trends Plant Sci 12 (8), 352-7.

34. Oliver, S.N. et al. (2009) Vernalization-induced flowering in cereals is associated with changes in histone methylation at the VERNALIZATION1 gene. Proc Natl Acad Sci U S A 106 (20), 8386-91. 35. Aravind, L. et al. (2003) Plasmodium biology: genomic gleanings. Cell 115 (7), 771-85. 36. Bischoff, E. and Vaquero, C. (2010) In silico and biological survey of transcription-associated proteins implicated in the transcriptional machinery during the erythrocytic development of Plasmodium falciparum. BMC Genomics 11, 34.

37. Kafsack, B.F. et al. (2014) A transcriptional switch underlies commitment to sexual development in malaria parasites. Nature 507 (7491), 248-52.

38. Sinha, A. et al. (2014) A cascade of DNA-binding proteins for sexual commitment and development in Plasmodium. Nature 507 (7491), 253-7.

39. Voorberg-van der Wel, A. et al. (2017) A comparative transcriptomic analysis of replicating and dormant liver stages of the relapsing malaria parasite Plasmodium cynomolgi. Elife 6.

40. Gural, N. et al. (2018) In Vitro Culture, Drug Sensitivity, and Transcriptome of Plasmodium Vivax Hypnozoites. Cell Host Microbe 23 (3), 395-406 e4.

41. Dembele, L. et al. (2014) Persistence and activation of malaria hypnozoites in long-term primary hepatocyte cultures. Nat Med 20 (3), 307-12.

42. Carlton, J.M. et al. (2008) Comparative genomics of the neglected human malaria parasite Plasmodium vivax. Nature 455 (7214), 757-63.

43. Tachibana, S. et al. (2012) Plasmodium cynomolgi genome sequences provide insight into Plasmodium vivax and the monkey malaria clade. Nat Genet 44 (9), 1051-5.

44. Charon, J. et al. (2019) Novel RNA viruses associated with Plasmodium vivax in human malaria and Leucocytozoon parasites in avian disease. PLoS Pathog 15 (12), e1008216. 
45. Zhang, M. et al. (2018) Uncovering the essential genes of the human malaria parasite Plasmodium falciparum by saturation mutagenesis. Science 360 (6388). 46. Howick, V.M. et al. (2019) The Malaria Cell Atlas: Single parasite transcriptomes across the complete Plasmodium life cycle. Science 365 (6455).

47. Sa, J.M. et al. (2020) Single-cell transcription analysis of Plasmodium vivax blood-stage parasites identifies stage- and species-specific profiles of expression. PLoS Biol 18 (5), e3000711. 48. Voorberg-van der Wel, A. et al. (2013) Transgenic fluorescent Plasmodium cynomolgi liver stages enable live imaging and purification of Malaria hypnozoite-forms. PLoS One 8 (1), e54888. 49. Dembele, L. et al. (2011) Towards an in vitro model of Plasmodium hypnozoites suitable for drug discovery. PLoS One 6 (3), e18162.

50. Cubi, R. et al. (2017) Laser capture microdissection enables transcriptomic analysis of dividing and quiescent liver stages of Plasmodium relapsing species. Cell Microbiol 19 (8).

51. March, S. et al. (2013) A microscale human liver platform that supports the hepatic stages of Plasmodium falciparum and vivax. Cell Host Microbe 14 (1), 104-15.

\section{Glossary}

APETALA-2 (ApiAP2): a group of transcription factors originally found in plants (AP2), and subsequently also in apicomplexans, hence 'Apicomplexan APETALA-2' OR 'ApiAP2'. ApiAP2 genes regulate many of the lifecycle transitions in Plasmodium parasites. In plants, AP2 factors also regulate developmental pathways, although they do not have a central role in vernalisation.

Convergent evolution: independent evolution of functionally similar traits in organisms that are not closely related and do not share the trait in their last common ancestor. Such 
evolution usually occurs in response to similar evolutionary pressures experienced by different organisms.

Hypnozoite: a dormant, non-replicating form of Plasmodium parasite that can occur inside liver cells after invasion by sporozoites of the species $P$. vivax, $P$. ovale curtisi or $P$. ovale wallikeri.

MADS-box: the protein motif that defines a group of transcription factors found widely in eukaryotic organisms, where they regulate developmental pathways. The name is an acronym of the first four MADS-box factors discovered (S. cerevisiae MINICHROMOSOME MAINTENANCE FACTOR 1, Arabidopsis thaliana AGAMOUS, Antirrhinum majus DEFICIENS and Homo sapiens Serum Response Factor).

Malariotherapy: the practise of deliberately infecting syphilis patients with malaria parasites to induce high fevers. These fevers could kill the causative Treponema pallidum bacterium, thus effecting a cure of the otherwise-terminal syphilitic disease.

Quantitative trait locus (QTL): a chromosomal region containing gene(s) that affect a 'quantitative trait', i.e. a phenotypic trait that varies in strength.

Radical cure: complete elimination of malaria parasites from an infected host - specifically elimination of dormant hypnozoites in the liver, thus preventing relapses.

422 Vernalisation: the induction of flowering in a vegetatively-growing plant (or an ungerminated seed) through exposure to prolonged cold temperatures, as would usually be experienced during winter. 
Within the past $\sim 7$ years, reports have begun to emerge on the molecular biology of the elusive hypnozoite. 2013 saw the first evidence that hypnozoites of $P$. cynomolgi, a sister species of $P$. vivax, could be established in macaque primary hepatocytes by infecting them with sporozoites bearing a fluorescent marker [48]. FACS could then be used to isolate both hypnozoites and actively-growing hepatic schizonts (cells that had not gone dormant after the infection), and drugs could be used to enrich for hypnozoites [49]. This paved the way for two transcriptomes from hypnozoites 6 and 9 days old [23,39]. Compared to hepatic schizonts, transcription in hypnozoites was dramatically and progressively downregulated. Genes remaining active included those involved in epigenetics, chromatin maintenance, ATP homeostasis and protein export. However, very few genes were specifically upregulated and there was no clear 'hypnozoite factor', although several members of the APETALA-2 (ApiAP2) family of apicomplexan-specific transcription factors were detected. Due to the lack of molecular genetics in $P$. vivax, it is not currently possible to replicate such studies using transgenic $P$. vivax.

A second group showed in 2014 that $P$. cynomolgi hypnozoites cultured for a period of several weeks could display reactivation, promoted by a histone methyltransferase inhibitor. This directly implicated epigenetics for the first time [41]. The group also produced a hypnozoite transcriptome [50] and posited a hypnozoite-specific $A P 2$ factor, but this was not reproduced by other groups $[23,39,40]$.

447 liver organoids, and again produced a transcriptome [40,51]. This recapitulated the general transcriptional shutdown described above. A single AP2 gene (PVP01_0916300) was 
somewhat upregulated in these hypnozoites, and was also found in $P$. cynomolgi hypnozoites [23], so it is currently the best candidate for a hypnozoite transcription factor. However, it is not unique to this lifecycle stage, being strongly expressed in other stages too, including gametocytes - another quiescent stage, but very different from hypnozoites. Importantly, none of these studies have identified a clear 'reactivation factor': a gene that might be switched off in hypnozoites but specifically upregulated in reactivating schizonts.

\section{Figure legends}

Figure 1. The lifecycle of Plasmodium parasites. A general Plasmodium lifecycle is shown schematically, highlighting the two distinct forms of hepatic parasite that can form in $P$. vivax and $P$. ovale - hypnozoites and hepatic schizonts. Sporozoites migrate to the liver from the site of a mosquito bite and invade hepatocytes: here, they either become dormant hypnozoites or immediately start to multiply asexually inside the host hepatocyte. In nonrelapsing parasite species, this latter is the only pathway believed to occur. After about a week of asexual growth, the resultant hepatic schizont releases thousands of merozoites, which then infect erythrocytes. In erythrocytes, repeated cycles of asexual replication, cell lysis and reinvasion occur, causing all the symptoms of malaria. A minority of these parasites differentiates into pre-gametes called gametocytes which circulate in a quiescent state ready for mosquito transmission. If taken up by a mosquito vector, the sexual cycle ensues, culminating in sporozoites that are injected into another human host during a mosquito bite. 
Figure 2. Lifecycle variation in strains of $\boldsymbol{P}$. vivax and $\boldsymbol{A}$. thaliana. (A) Schematic showing the fate of parasites after inoculation via mosquito bite in representative tropical, subtropical and temperate strains of $P$. vivax. Adapted from [8]. (B) Schematic lifecycles of two representative strains of $A$. thaliana - vernalising and fast-cycling.

\section{Figure 3. Molecular pathways in plant vernalisation and modelled pathways for}

hypnozoite dormancy. (A) The main factors controlling vernalisation in A. thaliana are shown. FLC, the repressor of flowering FLOWERING LOCUS C; FRI, the DNA-binding protein FRIGIDA; FT, a representative gene for flowering FLOWERING TIME; VIN3, the plant homeodomain protein VERNALISATION INSENSITIVE 3; VRN2, the polycomb-like protein VERNALISATION 2. (B) The main factors controlling vernalisation in cereals are shown. VRN1, the activator of flowering VERNALISATION 1; VRN2, the DNA-binding protein VERNALISATION 2 (unrelated to VRN2 in A. thaliana). (C) Schematic model for hypnozoite dynamics controlled by a putative ApiAP2 gene that promotes growth, ' $A P 2-H$ '. The gene is silenced by default in inoculated parasites that invade hepatocytes and become hypnozoites. Gene silencing decays slowly but can be accelerated by fever stimuli. When de-silenced, the AP2 factor activates genes for replication and growth, prompting hypnozoites to become hepatic schizonts and initiate an erythrocytic infection. The resultant malarial fever can then set the clock for future hypnozoite reactivations. (D) Schematic model for hypnozoite dynamics controlled by a putative ApiAP2 gene for dormancy. The model operates oppositely to that in panel C: the AP2 factor is actively transcribed by default and represses replication genes. Epigenetic silencing of $A P 2-H$ accumulates slowly and can be accelerated by fever stimuli. Once silenced, the AP2 factor 
495 schizonts and initiate an erythrocytic infection.

496 
Figure 1

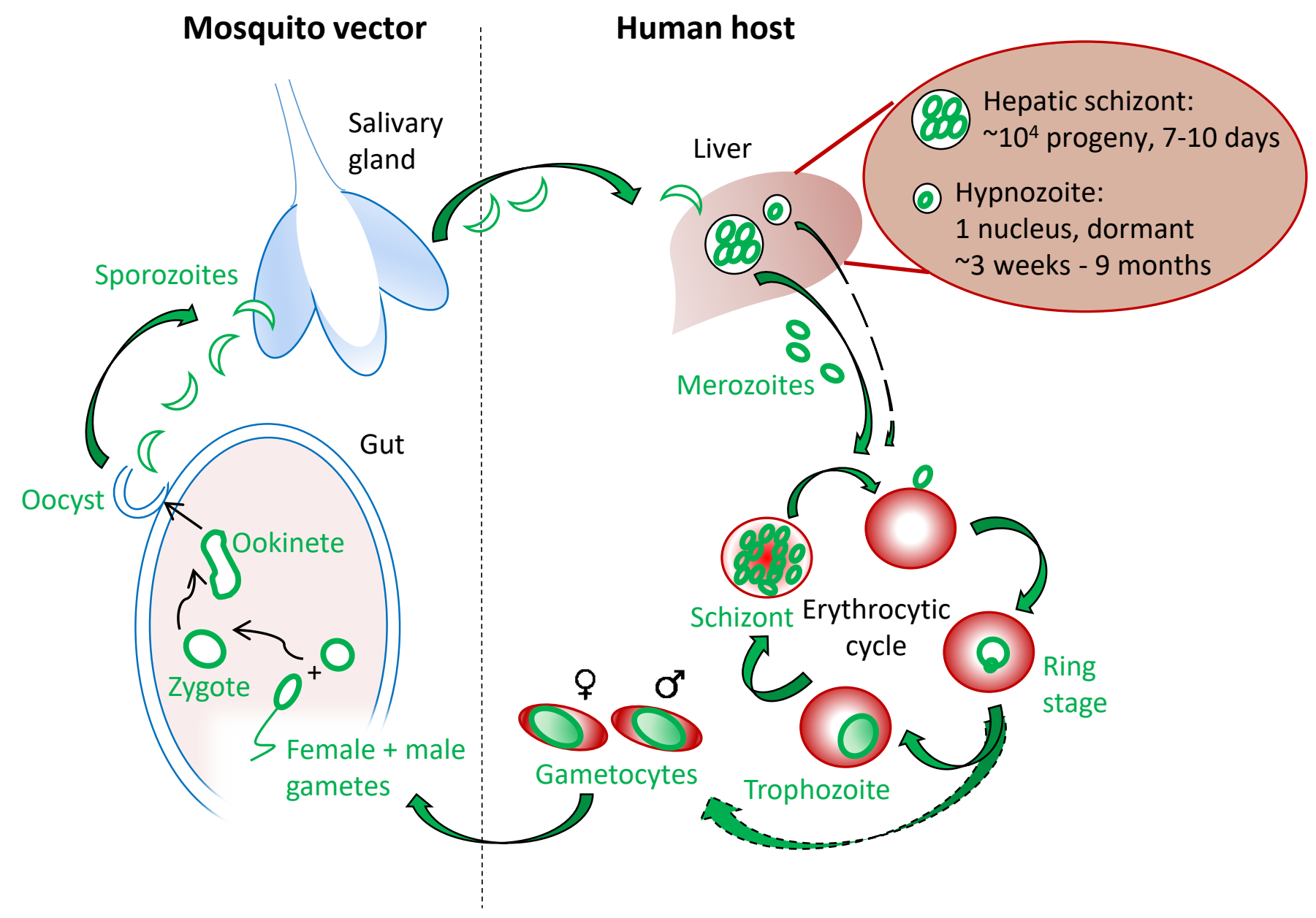



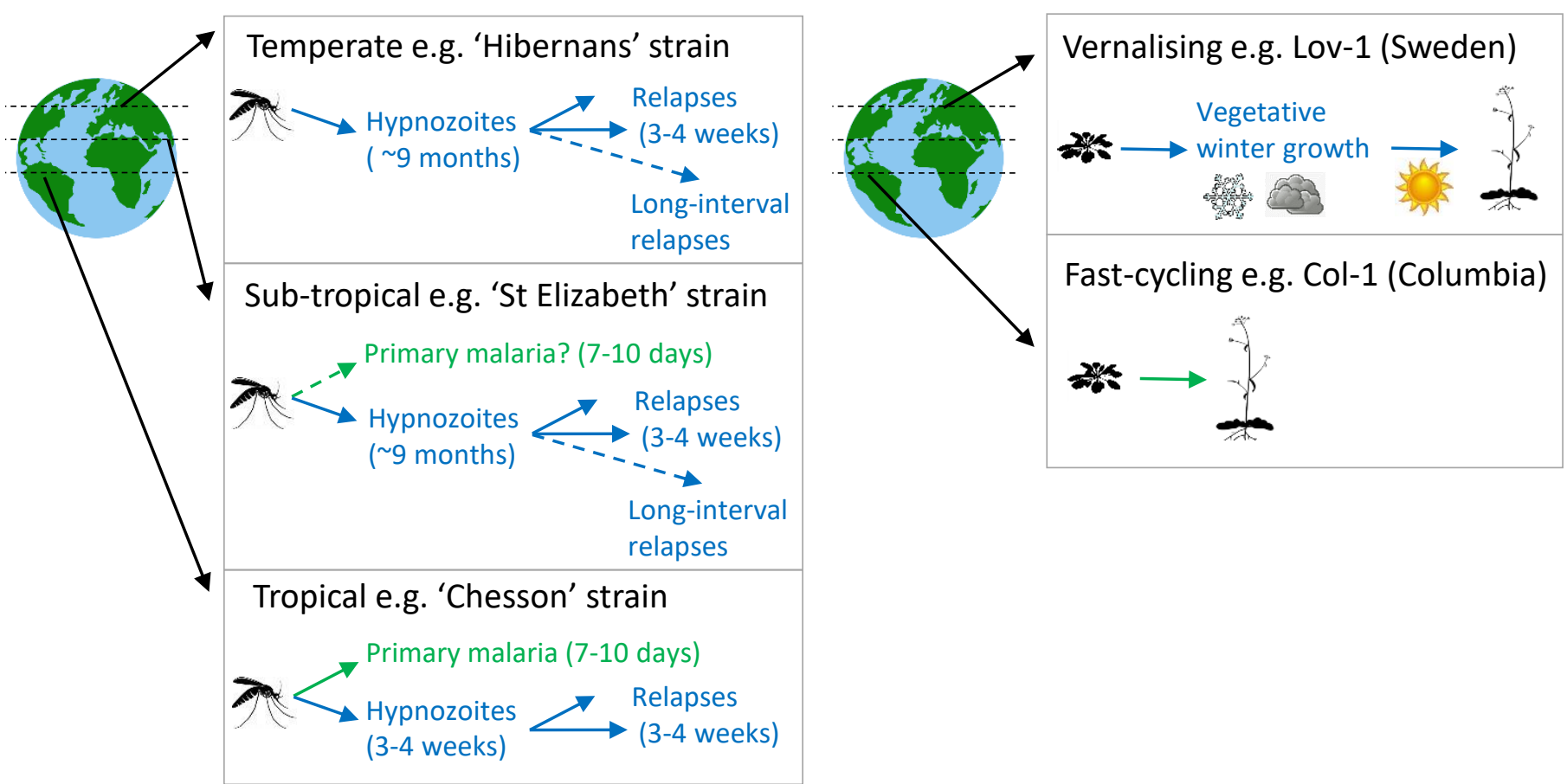


\section{A Arabidopsis}

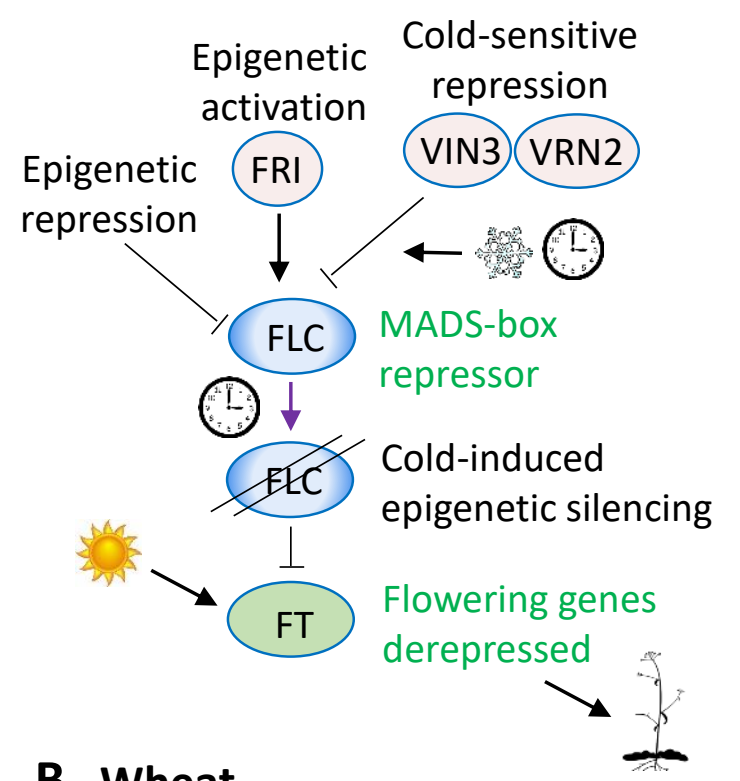

\section{B Wheat}

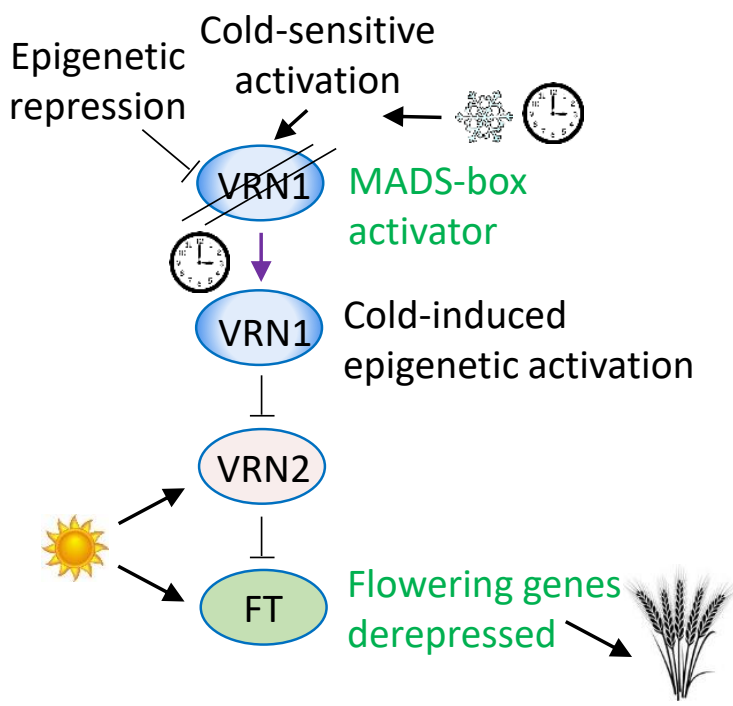

\section{P. vivax model: AP2-H for growth} Sub-tropical strain Tropical strain

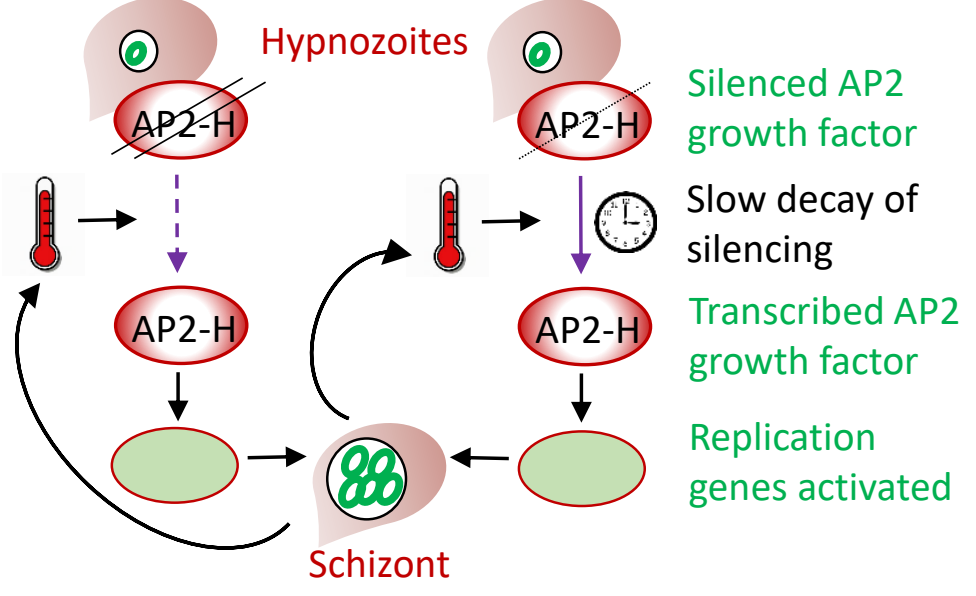

D P. vivax model: AP2-H for dormancy Sub-tropical strain Tropical strain

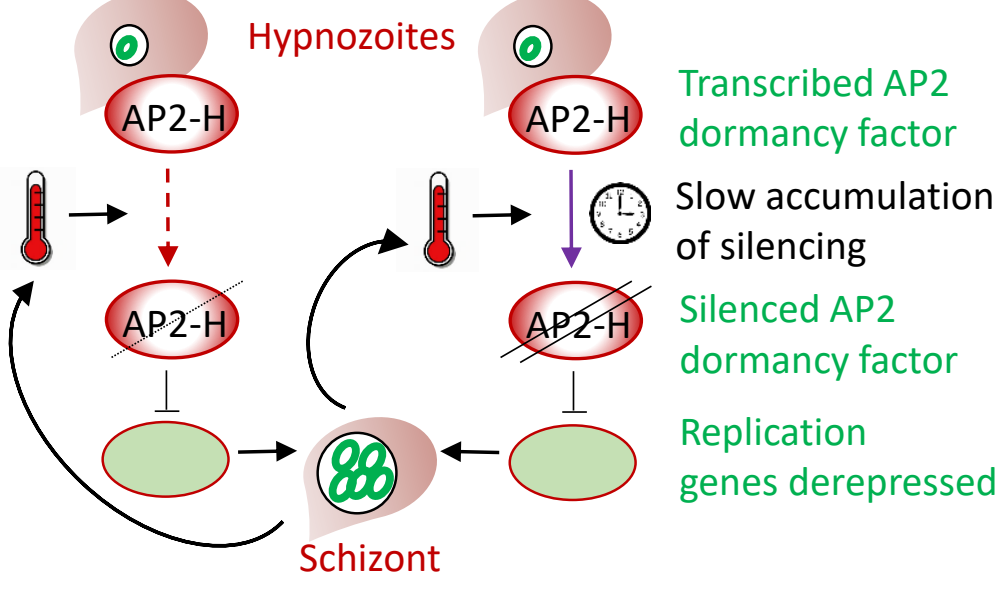


1

2

3

4

5

6

7

8

9

10

11

12

13

\section{Highlights}

- Hypnozoites are dormant malaria parasites that can reactivate and cause relapses of malarial disease. They are an important factor in malaria biology and in the feasibility of malaria elimination programmes.

- The behaviour of Plasmodium hypnozoites has been documented clinically but the molecular-genetic basis of this behaviour is entirely unknown.

- Hypnozoites appear to have an intrinsic 'reactivation clock', encoded genetically or epigenetically, yet also sensitive to certain stimuli from the mammalian host.

- The hypnozoite 'clock' bears striking similarities to the phenomenon of vernalisation in flowering plants, which is well-characterised on a molecular-genetic level.

- The molecular biology of vernalisation can be used to build a conceptual model for the pathways that could underlie hypnozoite dormancy and reactivation. 


\section{Outstanding Questions}

- What genes are involved in hypnozoite dormancy and reactivation? Is there a central regulator in the form of an ApiAP2 gene?

- Is the 'reactivation clock' based on a slowly-changing epigenetic signature, placed on an ApiAP2 or other gene(s)? If so, what factors might influence the rate of change in the epigenetic signature?

- Can the technology for transcriptomics from low cell numbers, or from single cells, be improved sufficiently to identify clear, reproducible genes whose transcription varies diagnostically in hypnozoites versus hepatic schizonts?

- Can epitranscriptomics be conducted at sufficient resolution on low cell numbers or single cells to define the epigenetic marks on such variantly-transcribed genes?

- Can molecular-genetic techniques be developed or improved sufficiently in relapsing malaria species ( $P$. vivax, P.ovale spp., or the primate model $P$. cynomolgi) to investigate any putative hypnozoite genes by reverse genetic experiments?

- Is the molecular basis for hypnozoite formation conserved in $P$. vivax and $P$. ovale spp., or has it evolved convergently in these species?

- Might viral genomes found in P. vivax (and possibly yet to be found in P. ovale) somehow confer the virus-like properties of dormancy and reactivation on the hepatic stages of these parasites?

- Are there shared factors between the molecular basis for hypnozoite dormancy and the molecular basis for the shorter-term quiescence that occurs in mature gametocytes? 